(4) थ/Ascofapsi

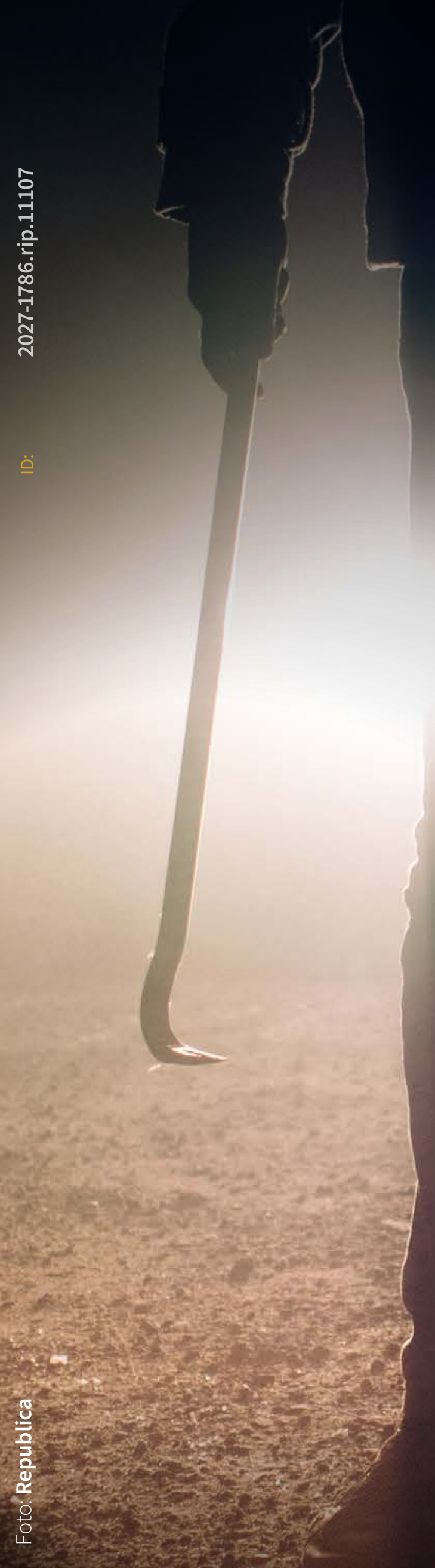

\title{
Ocho factores de la conducta

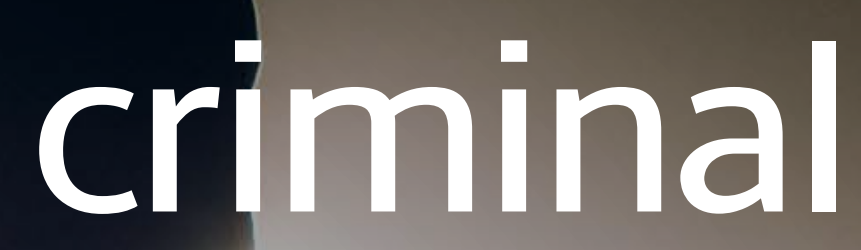

Aplicabilidad en jóvenes mexicanos

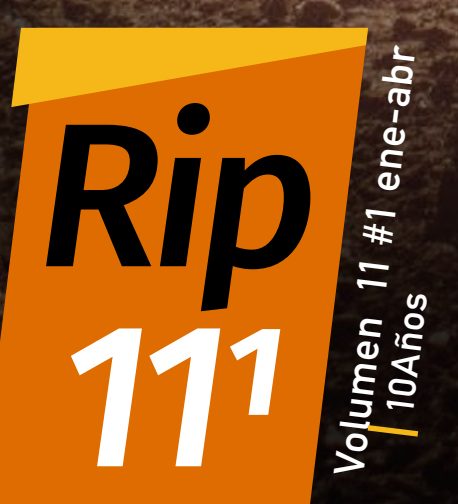

The 8 factors of criminal behavior: applicability in young Mexicans

(c) $\underset{\mathrm{BY}}{(i)}$

Julio Isaac Vega Cauich Graciela Mayte Chale Cervantes Aaron Javier Euan Catzin
Cindy Carolina Cauich Sonda
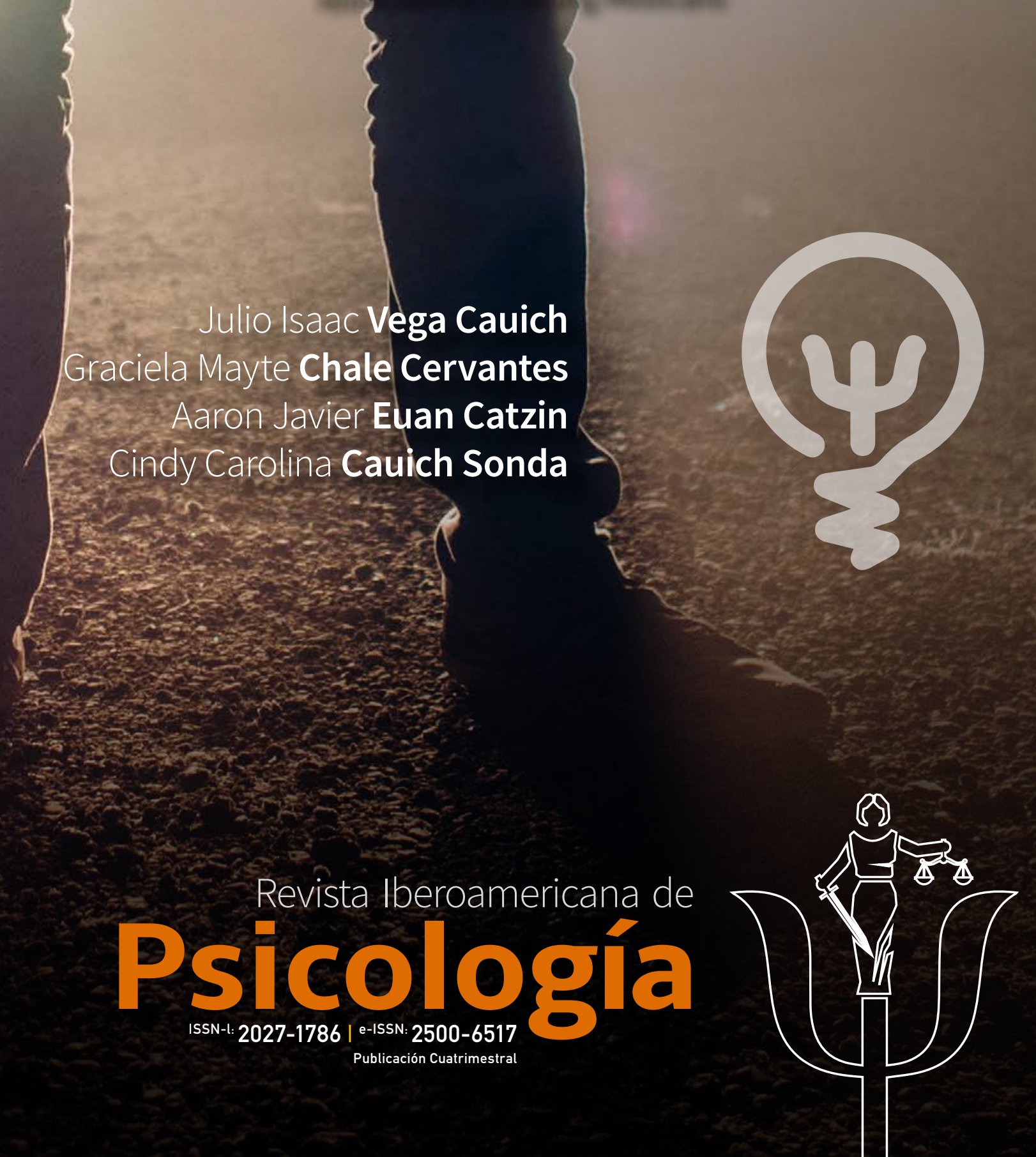
Revista Iberoamericana de

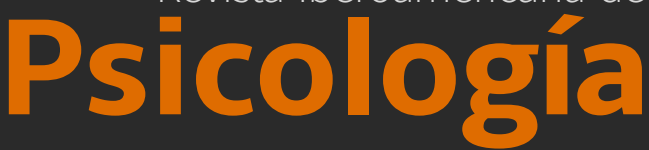

ISSN-I: 2027-1786 | e-ISSN: 2500-6517 Publicación Cuatrimestral

ID:

2027-1786.rip.11107

Title: $\quad$ The 8 factors of criminal behavior

Subtitle: Applicability in young mexicans

Título: Ocho factores de la conducta criminal

Subtítulo: Aplicabilidad en jóvenes mexicanos

Alt Title / Título alternativo:

[en]: Applicability of the central Eight Factors Model of Criminal Behavior in young Mexicans

[es]: $\quad$ Aplicabilidad del modelo de los ocho grandes factores de la conducta criminal en jóvenes mexicanos

Author (s) / Autor (es):

Vega Cauich, Chale Cervantes, Euan Catzin, \& Cauich Sonda

Keywords / Palabras Clave:

risk factors; antisocial behavior; juvenile delinquency; risk assessment

[es]

factores de riesgo; conducta antisocial; delincuencia juvenil; valoración del riesgo

Submited: 2017-12-15

Acepted: 2018-03-23

\section{Resumen}

Objetivo: Identificar cuáles de los ocho factores de riesgo del modelo de riesgo-necesidadresponsividad (RNR) de Andrews y Bonta (2010) son mejores predictores de la conducta delictiva en los jóvenes mexicanos. Método: Fueron seleccionadas variables de la Encuesta de Cohesión Social para la Prevención de la Violencia y la Delincuencia (ECOPRED, 2014) que presentaran relación con los ocho factores de riesgo, las cuales fueron sintetizadas mediante un análisis factorial. Posteriormente, se llevó a cabo una regresión logística binaria, la variable de respuesta fue haber tenido o no un arresto policial en lo que iba del año, lo cual permitió generar un modelo con mejor capacidad para predecir si los jóvenes presentarían arrestos. Resultados: En el modelo generado interactúan cinco de los ocho factores de riesgo: historial de conducta antisocial, asociación criminal, abuso de sustancias, pobres relaciones familiares, y problemas en el trabajo y la escuela. Discusión: Los resultados obtenidos coinciden con estudios realizados en otros países, en los que los ocho factores

del modelo RNR no siempre se presentan

en población juvenil, ya que existen factores (como la edad, la cultura, el sexo o la geografía) que interactúan con las variables, provocando el aumento o la disminución de su influencia en la conducta delictiva.

\section{Abstract}

Objective: Identify which of the eight risk factors from Andrews and Bonta's Risk-Need-

Responsivity (RNR) model (2010) are better predictors of criminal behavior in Mexican youth. Method: Variables from the Social Cohesion Survey for the Prevention of Violence and Crime (ECOPRED, 2014) were selected

which related to the eight risk factors of

Andrews and Bonta's RNR. These variables were synthesized by a factorial analysis. A binary logistic regression was carried out, the response variable was "having or not having a police arrest in the current year", which allowed

generating a model with a better capacity to predict if young people would undergo arrests. Results: In the generated model, five of the eight risk factors interact: a history of antisocial behavior, criminal association, substance abuse, poor family relationships, and problems at work and at school. Discussion:

The obtained results show similarities with studies carried out in countries, in which the eight factors of the RNR model not always show

up in the juvenile population, since there are factors (like age, culture, sex, or geography) that interact with the variables causing the increase or decrease of their influence on criminal behavior.

\author{
Lic Julio Isaac Vega Cauich, [MSc] Psi sp \\ ORCID: $\quad$ 0000-0001-9190-3720 \\ e-mail: julio.vega@outlook.com \\ Lic Graciela Mayte Chale Cervantes, MSc Ps \\ ORCID: $\quad$ 0000-0003-1042-4475 \\ e-mail: $\quad$ psic.gracielachale@gmail.com \\ Lic Aaron Javier Euan Catzin, Psisp \\ ORCID: $\quad$ 0000-0001-6119-0352 \\ e-mail: $\quad$ euan.aaron@outlook.com \\ Lic Cindy Carolina Cauich Sonda, MSc Psi \\ ORCID: 0000-0003-0447-6658 \\ e-mail: $\quad$ cindycaro.cs@gmail.com
}

Source |Filiacion:

Foco Rojo: Centro de Psicología Aplicada

$\mathrm{BIO}$ :

(NOTA: La información biográfica de los autores se detalla al final del artículo).

City | Ciudad:

México DF $[\mathrm{mx}]$

Citar como:

Vega Cauich, J. I. , Chale Cervantes, G. M., Euan Catzin, A. J., \& Cauich Sonda, C. C. (2018). Ocho factores de la conducta criminal: Aplicabilidad en jóvenes mexicanos. Revista Iberoamericana de Psicología issn-l:2027-1786, 11 (1), 65-76. Obtenido de: https://revistas.iberoamericana.edu.co/index.php/ripsicologia/article/view/1311 


\title{
Ocho factores de la conducta criminal Aplicabilidad en jóvenes mexicanos
}

\author{
The 8 factors of criminal behavior: applicability in young Mexicans \\ Julio Isaac Vega Cauich \\ Graciela Mayte Chale Cervantes \\ Aaron Javier Euan Catzin \\ Cindy Carolina Cauich Sonda
}

\section{Introducción}

Durante el año 2015, en México, los juzgados especializados en adolescentes abrieron 10,647 casos, de los cuales el 89.7\% recibieron una medida sancionatoria (en tanto los adolescentes en cuestión fueron encontrados responsables de haber cometido un delito), mientras que en tan solo el 10.3\% de los casos los adolescentes fueron declarados inocentes (Inegi, 2016). Este aumento en la tasa delictiva juvenil plantea el reto de identificar los factores de riesgo de conducta delictiva en la población adolescente mexicana, con miras a abordarlos, lograr una mejor prevención e intervención y así, reducir esas tasas.

La Oficina de las Naciones Unidas contra la Droga y el Delito (Unodc, 2013), platea que: "La prevención de conducta delictiva y la reincidencia, requiere intervenciones efectivas basadas en la comprensión de los factores que representan un riesgo para los delincuentes y [que] les dificultan el éxito de su reintegración en la sociedad" (pág. 11). En consonancia lo con anteriormente mencionado, el Modelo RiesgoNecesidad-Responsividad (RNR) desarrollado por Andrews y Bonta permite identificar factores de riesgo empíricamente asociados a la conducta criminal, y ha probado ser eficaz en diferentes partes del mundo en lo que respecta a la evaluación y tratamiento de adolescentes quienes han cometido un delito. Dicho modelo parte fundamentalmente de la premisa que la identificación temprana y la prevención de los factores de riesgo evita que los jóvenes inicien en la conducta delictiva (Hoge, 2015). Las acciones preventivas que han demostrado mayor eficacia, además de ser divertidas para los jóvenes (Wilson \& Hoge, 2013), están relacionadas con intervenciones que promueven la interacción prosocial con otros miembros de la comunidad en la que el individuo se desenvuelve, pues no puede se puede dejar de lado la influencia que tiene la cultura en los factores propuestos por este modelo (Hoge, 2015). Es por este engranaje que la delincuencia juvenil es considerada un fenómeno multifactorial que abarca aspectos sociales, psicológicos, biológicos y de la historia de vida (Hoge, 2015).
La importancia del presente estudio radica en la propuesta de acciones efectivas de prevención de factores de riesgo para evitar que los jóvenes mexicanos inicien en conductas delictivas y, en el caso de jóvenes quienes ya presenten problemas con la ley, en el diseño individualizado de intervenciones adecuadas cuyas estrategias incidan directamente sobre los factores relacionados de forma directa con el fin de evitar la reincidencia delictiva de estos jóvenes.

\section{Marco teórico}

En la década de los 90, los autores Andrews y Bonta desarrollaron el denominado Modelo de Riesgo-Necesidad-Responsividad (RNR), que tiene sus bases en la teoría general de la personalidad y en la teoría del aprendizaje cognitivo social de la conducta delictiva (Bonta \& Andrews, 2012). Este modelo de intervención para infractores de ley destaca por el uso de herramientas, tecnologías y metodologías existentes relacionadas con la conducta delictiva y ha demostrado su efectividad empíricamente (Velásquez Valenzuela, 2014), por lo que no es de extrañar que se recurra a éste para fundamentar tanto acciones de prevención del delito como el tratamiento de infractores adultos y adolescentes. Su importancia ha sido reconocida en todo el mundo y se ha traducido a varios idiomas y, a pesar de ser un modelo canadiense, su aplicación en población latinoamericana (Montedónico Godoy, 2015) ha sido demostrada.

Dicho modelo está compuesto por tres ejes principales que le dan el nombre al mismo (Andrews \& Bonta, 2010; James, 2015):

1. el principio de riesgo (cuya premisa es que la conducta criminal puede se puede predecir, que el tratamiento se debe focalizar en los casos de alto riesgo y que la reincidencia se puede reducir si el tratamiento brindado es proporcional al riesgo de reincidencia

2. el principio de necesidad (que destaca la importancia de reducir las necesidades criminógenas y no otras)

3. el principio de responsividad (que describe la forma como se debe proporcionar el tratamiento dependiendo del estilo de aprendizaje, motivación, habilidades y fortalezas del infractor). 


\section{Ocho factores de la conducta criminal}

Aplicabilidad en jóvenes mexicanos

Al plantear por primera vez el modelo, los autores identificaron los Big Four, es decir, cuatro grandes factores asociados con la conducta delictiva, a saber: historial de conducta criminal, rasgos de personalidad antisocial, pensamiento antisocial y asociación antisocial (Andrews \& Bonta, 2010). Posteriormente le sumaron otros cuatro factores con capacidad predictiva moderada: abuso de sustancias, relaciones familiares y de pareja, uso del tiempo libre y situación escolar y laboral (Grieger \& Hosser, 2014; Nguyen, Arbach-Lucioni, \& Andrés-Pueyo, 2011). Así entonces, el modelo contempla ocho grandes factores (Big Eight), que incluyen tanto factores de riesgo estáticos como dinámicos (James, 2015).

\section{Los ocho principales factores de Riesgo-Necesidad- Responsividad}

Como se ha mencionado, este modelo propone ocho factores asociados con la conducta delictiva (cuatro de riesgo alto y cuatro de riesgo moderado) que no solamente aumentan el riesgo que una persona comience y mantenga una conducta criminal, sino que también describen las necesidades que deberían guiar las propuestas de tratamiento en el sistema penitenciario (Andrews \& Bonta, 2010; Looman \& Abracen, 2013). Basados en los trabajos de Andrews y Bonta (2010), James (2015), Looman y Abracen (2013), estos factores son descritos a continuación de manera breve. También se hará referencia a algunos estudios que han mostrado una relación entre éstos y la conducta delictiva o la reincidencia en jóvenes y adolescentes:

\section{Historial de conducta antisocial}

Relacionado con el temprano involucramiento en conductas antisociales varias, por lo que contempla ser arrestado a corta edad, la violación a las reglas de libertad condicional y el número de delitos o conductas antisociales. Al respecto, las detenciones policiales previas y las agresiones marcan una diferencia significativa al comparar grupos de adolescentes con problemas con la ley y aquellos sin problemas (Herrera, Rueda, \& Martínez, 2013). Estudios indican que los antecedentes delictivos están relacionados con la reincidencia (Assink, y otros, 2015; Bertone, Domínguez, Vallejos, Muniello, \& López, 2013; Martínez-Catena \& Redondo, 2013; Ortega Campos, García, de la Fuente Sánchez, \& Zaldívar Basurto, 2012; PérezLuco, Lagos, \& Báez, 2012).

\section{Patrones de personalidad antisocial}

Asociado con la impulsividad, la búsqueda constante de emociones y placer, desprecio por los demás y comportamiento agresivo. Los riesgos asociados se deben a un autocontrol débil, mal manejo de la ira y pobres habilidades para la resolución de problemas. Al respecto, investigaciones han demostrado que los adolescentes quienes tienen un bajo autocontrol ante el conflicto (como actitudes agresivas o respuestas impulsivas) presentan un mayor riesgo de reincidencia delictiva (Assink, y otros, 2015; Farrington, Gaffney, \& Ttofi, 2017; Jolliffe, Farrington, Piquero, Loeber, \& Hill, 2017; Navarro-Pérez \& Pastor-Seller, 2017; Pelegrín \& Garcés de los Fayos, 2008; Rodríguez, Mirón, \& Rial, 2012).

\section{Pensamiento antisocial}

Las actitudes o pensamientos pro-criminales refieren a aquellas actitudes que apoyan la conducta criminal y delictiva (Andrews \& Bonta, 2010), y suelen expresarse en tres formas: justificación de la violencia, identificación criminal (como el admirar a criminales) y rechazo de la autoridad (Andrews \& Bonta, 2010).

En un estudio realizado en España, Rojas Zerega (Rojas Zegarra, 2013) encontró que las distorsiones cognitivas auto-sirvientes se relacionan con la agresión y las conductas delictivas. Esta autora define dichas distorsiones como aquellos patrones cognitivos sesgados que pueden iniciar, facilitar o perpetuar las tendencias antisociales, en tanto promueven la autojustificación cuando se transgreden normas morales.

En general, la evidencia empírica soporta la consistencia entre los pensamientos pro-criminales y la reincidencia (Assink, y otros, 2015; Banse, Koppehele-Gossel, Kistemaker, Werner, \& Schmidt, 2013), elementos que a su vez están interrelacionados con la influencia de los pares antisociales (Boduszek, Hyland, Pedziszczak, \& Kielkiewicz, 2012).

Lamentablemente la intervención con delincuentes, que busca reducir estas actitudes y pensamientos pro-criminales, no ha demostrado ser tan efectiva como lo son otras formas de intervención (Banse, Koppehele-Gossel, Kistemaker, Werner, \& Schmidt, 2013).

\section{Asociaciones delictivas o antisociales}

Se presentan cuando el sujeto se relaciona con personas cuya conducta es antisocial y, a su vez, se aparta de aquellos quienes tienen conductas prosociales. Esta relación o asociación debe presentarse con personas quienes resultan importantes para el individuo. Estudios muestran que tener un grupo de amigos que lleve a cabo conductas antisociales es un factor de riesgo fundamental para los adolescentes en lo que respecta a cometer actos de este tipo (Benedicto, 2015; NavarroPérez \& Pastor-Seller, 2017; Ortega-Campos, García-García, \& Frías-Armenta, 2014; Rodriguez, 2015; Rodríguez, Mirón, \& Rial, 2012; Schioppa, 2013). Al respecto, en su trabajo, Cuervo, Villanueva y Prado-Gascó (2017) encontraron que este factor era el mejor predictor de la reincidencia delictiva en la muestra de adolescentes.

\section{Situaciones problemáticas en el hogar}

Refiere a la baja calidad de las relaciones entre el hijo/hija con sus padres, o entre la pareja (sean estas relaciones matrimoniales o no) y también contempla la promoción de posturas pro-criminales por parte de la familia o pareja, o la permisividad ante conductas antisociales.

En el modelo propuesto por Rodríguez, Mirron y Rial (2012), se encontró que la inadecuada supervisión familiar es un factor que aumenta las probabilidades que adolescentes presenten conductas antisociales, resultados que son consistentes con los encontrados por Schioppa (2013) y Martínez, Fuentes, García y Madrid (2013), sobre el control parental y cometer delitos. Otros estudios han revelado una relación entre violencia familiar, problemas físicos y antecedentes penales en la familia, (Herrera, Rueda, \& Martínez, 2013; Ortega-Campos, GarcíaGarcía, \& Frías-Armenta, 2014; Ortega-Campos, García-García, \& Frías-Armenta, 2014; Rodríguez, Mirón, \& Rial, 2012), las relaciones familiares en general (Assink, y otros, 2015; Farrington, Gaffney, \& Ttofi, 2017; Jolliffe, Farrington, Piquero, Loeber, \& Hill, 2017) como factores de riesgo de reincidencia delictiva en los adolescentes.

\section{Problemas en la escuela o trabajo}

Se relaciona con bajos niveles de desempeño, satisfacción o expectativas relacionadas con la actividad escolar o el trabajo que se lleva a cabo. 
Algunos estudios revelan que adolescentes en situaciones educativas negativas (como no contar con redes de educación formales y/o informales) (Navarro-Pérez \& Pastor-Seller, 2017), tener bajo nivel educativo (Bertone, Domínguez, Vallejos, Muniello, \& López, 2013), así como problemas escolares en general) tienen un mayor riesgo de cometer conductas antisociales (Cuervo, Villanueva, \& Prado-Gascó, 2017; Farrington, Gaffney, \& Ttofi, 2017; Herrera, Rueda, \& Martínez, 2013; Pelegrín \& Garcés de los Fayos, 2008). Por otro lado, los problemas relacionados con el trabajo (como el pobre desempleo y la falta de redes sociales en este ámbito) también son factores que se han asociado a este tipo de conductas (Jolliffe, Farrington, Piquero, Loeber, \& Hill, 2017; Navarro-Pérez \& Pastor-Seller, 2017; Ortega Campos, García, de la Fuente Sánchez, \& Zaldívar Basurto, 2012).

\section{Pobre manejo positivo del tiempo libre}

Refiereal bajo involucramientoysatisfacción en actividades prosociales. Estudios han encontrado que la búsqueda de diversión relajante constituye una variable que diferencia a los adolescentes infractores de los no infractores (Vilariño, Amado, \&Alves, 2013), por ejemplo, en un estudio realizado en España se encontró que la práctica de deporte en familia funciona como una variable de prevención de problemas de conducta delictiva en adolescentes (Benavides, Romero, Fernández, \& Pichardo, 2017).

\section{Abuso de sustancias}

Refiere a problemas de abuso de alcohol u otras drogas que provocan dificultades en diferentes ámbitos. En general, estudios muestran que existe una relación significativa entre el consumo de sustancias y la conducta y reincidencia delictivas (Assink, y otros, 2015; Benedicto, 2015; Farrington, Gaffney, \& Ttofi, 2017; Herrera, Rueda, \& Martínez, 2013; Jolliffe, Farrington, Piquero, Loeber, \& Hill, 2017), y otros sugieren que la asociación de esta variable con la pertenencia a grupos fuera de la norma aumenta las probabilidades de cometer actos violentos (Contreras Martínez, Molina Banqueri,\&Cano Lozano,2012). En el caso de Latinoamérica, específicamente en Chile, Colombia y Perú, se observa una marcada relación entre el consumo de drogas y cometer delitos no violentos (Unodc, 2010).

\section{Otros aspectos relevantes}

En relación con estos factores resulta importante considerar el género, ya que existen diferencias significativas en cuanto a los factores de riesgo que presentan hombres y mujeres jóvenes quienes delinquen (Brogan, Haney-Caron, NeMoyer, \& DeMatteo, 2015; Thompson \& Morris, 2013). Ser hombre constituye como una variable predictora en tanto los hombres presentan mayor riesgo de reincidencia que las mujeres (Cuervo, Villanueva, \& Prado-Gascó, 2017; Ortega Campos, García, de la Fuente Sánchez, \& Zaldívar Basurto, 2012). Al respecto, Rodríguez, Mirrón y Rial (2012) y Moreira Trillo (2011) encontraron que, en los hombres, las variables familiares y escolares (en conjunto) guardan una relación significativa con el desarrollo de conductas antisociales. Específicamente la supervisión paterna parece jugar un papel protector para ellos, mientras que la pertenencia a grupos antisociales, así como el bajo nivel de autocontrol, resultan ser variables asociadas a conductas antisociales (Rodríguez, Mirón, \& Rial, 2012). Por último, el aprovechamiento académico también es un factor asociado de manera significativa a la delincuencia juvenil en hombres (Thompson \& Morris, 2013).

Sin embargo, es preciso aclarar que, a pesar de estas diferencias, en general los factores de riesgo siguen siendo consistentes en los casos de alto riesgo tanto en hombres como en mujeres (Andrews, y otros, 2012).
Queda de todo lo anterior que los factores hasta el momento mencionados se asocian de manera consistente con la delincuencia, por lo que no es de extrañar que existan estudios que analizan estos ocho factores de manera conjunta para predecir la delincuencia. Al respecto, el modelo propuesto por Andrews y Bonta ha mostrado resultados consistentes para predecir la reincidencia, tanto en adultos como en jóvenes (Brogan, Haney-Caron, NeMoyer, \& DeMatteo, 2015), sin embargo, los estudios con población de delincuentes juveniles han tenido mayor variabilidad en lo que refiere al aporte de cada factor.

Un estudio realizado en Australia por McGrath y Thompson (2012), con 3,658 delincuentes juveniles, reveló que no todos los factores considerados en el modelo de Andrewsy Bonta resultaban significativos para predecir la reincidencia penitenciaria. En su estudio se encontró que los factores familiares, las actividades recreativas prosociales y la personalidad antisocial no aportaban de forma significativa para predecir la delincuencia. Sin embargo, los otros cinco factores (historia de conducta antisocial, educación/trabajo, asociación delictiva, abuso de sustancias y actitudes pro-criminales) sí contribuían para explicar el $\mathbf{1 0 . 3}$ \% de la varianza de la delincuencia juvenil. Igualmente, en un estudio llevado a cabo por Grieger y Hosser (2014) en Alemania, con 589 delincuentes juveniles varones, se encontró que no todos los factores aportaban de manera significativa a la predicción de la reincidencia. En particular, el historial de comportamiento antisocial, los pensamientos pro-criminales, la escuela y el uso de sustancias eran significativos, pero no lo fueron la personalidad antisocial, las asociaciones criminales, la familia o las actividades recreativas. En otro estudio realizado en Singapur con 3,264 delincuentes juveniles de ambos sexos, Chu, Yu, Lee y Zeng (2014) encontraron que las asociaciones criminales, el abuso de sustancias y las actitudes pro-criminales no eran significativas para predecir la reincidencia, sin embargo, sí lo fueron el historial de conducta antisocial, la escuela/trabajo, las actividades recreativas, la personalidad antisocial y las relaciones familiares.

La gran disparidad de estos resultados posiblemente se deba a un fenómeno geográfico y cultural asociado a cada país donde se llevaron a cabo los estudios, pues el modelo original fue desarrollado en Canadá. Se ha visto que instrumentos que evalúan los ocho factores de Andrews y Bonta, como el Level of Service, tienden a mostrar resultados que varían dependiendo de la etnicidad e incluso la región geográfica (Olver, Stockdale, \& Wormith, 2014), mostrando mayores efectos en muestras procedentes de Canadá. A raíz de lo anterior, el presente estudio pretende validar el aporte de estos ocho factores en una muestra representativa de jóvenes mexicanos, con la finalidad de determinar la adecuación de los ocho factores en el contexto mexicano.

\section{Método}

Se realizó un estudio tipo observacional, retrospectivo y con alcance correlacional, con la finalidad de generar un modelo para predecir la reincidencia policial en adolescentes. Para ello fueron tomados en cuenta los factores de riesgo más asociados a la conducta criminal en jóvenes de México.

\section{Participantes.}

Para obtener la muestra final del presente estudio, se recurrió a la base de datos de la Encuesta de Cohesión Social para la Prevención de la Violencia y la Delincuencia (Ecopred), un proyecto de alcance nacional llevado a cabo por el Instituto Nacional de Estadística y 


\section{Aplicabilidad en jóvenes mexicanos}

Geografía (Inegi, 2014), cuya encuesta, bases de datos y resultados son publicados en la página del instituto para su acceso gratuito y libre. El proyecto general provee información de $\mathbf{8 4 , 9 2 8}$ cabezas de familia de todo el territorio mexicano, quienes comprendieron la primera parte del muestreo probabilístico estratificado con representatividad por cada estado de México. La segunda fase del muestreo provee información de $\mathbf{4 0 , 3 6 6}$ jóvenes de 12 a 29 años de edad de todo México. El presente estudio emplea precisamente ésta muestra de jóvenes, conformada en un $\mathbf{5 1 . 1 \%}$ por hombres y un $\mathbf{4 8 . 9 \%}$ mujeres, con edad promedio de $\mathbf{1 8 . 7}$ años y una desviación estándar de $\mathbf{4 . 7 5}$ años. De estos participantes, el 1.2\% $(n=490)$ mencionó haber sido arrestado por la policía alguna vez en el periodo que comprende el inicio del año en curso hasta la fecha de aplicación de la encuesta.

\section{Variables}

Dado que el estudio toma en consideración el modelo de los ocho grandes factores propuesto por Andrews y Bonta (2010), tres (3) jueces (psicólogos titulados con experiencia en la psicología jurídica) analizaron qué reactivos de la Ecopred evaluaban cada uno de los ocho factores propuestos por dicho modelo, con el fin de conservar aquellos reactivos aprobados de forma unánime por ellos.

Las variables conformadas por más de un reactivo (por ejemplo, estilos parentales de crianza o el índice de violencia familiar) habían sido previamente sintetizadas mediante un análisis factorial exploratorio que indicó la forma en la que se agrupaban los reactivos. El análisis factorial fue realizado con el método de componentes principales con rotación varimax, para evitar problemas de multicolinealidad en los análisis posteriores. Posteriormente, la consistencia interna de estos nuevos índices fue explorada mediante el estadístico Alfa de Cronbach para los índices formados por variables ordinales, y el estadístico KR-20 para los índices conformados por reactivos dicotómicos. Finalmente se crearon índices a partir de los reactivos de cada escala mediante la suma o promedio de sus ítems (Dawes \& Corrigan, 1974) y la consistencia interna de cada índice fue reportada.

En la Tabla 1 cada uno de los reactivos (o índices considerados) es descrito de manera detallada al igual que el factor que evalúan y la consistencia interna (en el caso de los índices creados).

Tabla 1 Especificación de los indicadores utilizados para cada factor propuesto en el modelo de Andrews y Bonta

\begin{tabular}{|c|c|c|}
\hline Factor & Reactivo o índice & Tipo de respuesta \\
\hline \multirow{3}{*}{$\begin{array}{l}\text { Historial de } \\
\text { conducta } \\
\text { antisocial }\end{array}$} & $\begin{array}{l}\text { Arresto en lo que va del año } \\
\text { (considerada como la variable de respuesta). }\end{array}$ & Nominal: Sí o No \\
\hline & Antecedentes de uso o portación de armas & Nominal: Sí o No \\
\hline & $\begin{array}{l}\text { Antecedentes de vandalismo } \\
\text { (grafitis, destrucción de propiedad ajena, etc.) }\end{array}$ & Nominal: Sí o No \\
\hline $\begin{array}{l}\text { Personalidad } \\
\text { Antisocial }\end{array}$ & $\begin{array}{l}\text { Impulsividad (índice formado a partir de reactivos que evalúan } \\
\text { control de impulsos). } \\
\text { Consistencia interna: } 0.63\end{array}$ & $\begin{array}{l}\text { Numérico. } \\
\text { Menor puntaje corresponde a } \\
\text { menor control de impulsos. }\end{array}$ \\
\hline \multirow{3}{*}{$\begin{array}{l}\text { Pensamientos } \\
\text { pro-criminales } \\
\text { (identificación } \\
\quad \text { criminal) }\end{array}$} & $\begin{array}{l}\text { Admira a un criminal o delincuente } \\
\text { (como asesino, secuestrador, etc.). }\end{array}$ & Nominal: Sí o No \\
\hline & Admira a un capo o líder de una banda violenta. & Nominal: Sí o No \\
\hline & Pares antisociales: Amigos cercanos con conductas antisociales & Numérica. \\
\hline
\end{tabular}

(índice formado por reactivos que evalúan si sus mejores amigos realizan conductas antisociales).

Compañeros de escuela/trabajo con actitudes antisociales (índice formado por reactivos que evalúan si sus compañeros de escuela o trabajo realizan conductas antisociales) Consistencia interna: 0.63

Antecedentes de pertenencia a bandas o pandillas

Número de sustancias que reporta haber consumido (índice Abuso de formado de la sumatoria aritmética del número de sustancias sustancias que afirma haber probado).

Menor puntaje significa menor número de conductas antisociales por parte de sus pares. Numérica.

Menor puntaje significa menor número de conductas antisociales por parte de sus compañeros.

Nominal: Sí o No Numérica.

Mayores puntajes significan experimentación con mayor número de sustancias. Ordinal.

¿Cómo se siente en casa?

1 (muy bien) a 4 (muy mal). Numérica.

Horas promedio entre semana de convivencia con los padres

Mayores puntajes corresponden a mayor cantidad de horas de convivencia.

Estilos parentales de crianza: índices conformados por los estilos parentales del padre y la madre. La madre consistió en dos índices (atención y control) y el padre de uno (atención/control). Atención de la madre: $\alpha=0.94$ Control de la madre: $\alpha=0.87$ Atención/Control del padre: $\alpha=0.96$

Relaciones familiares

Convivencia familiar. Índice conformado por reactivos que evalúa la convivencia con todos los miembros de la familia. Se obtuvo dos índices, uno sobre convivencia positiva y otro de convivencia negativa.

Convivencia positiva: $\alpha=0.64$ Convivencia negativa: $\alpha=0.57$

Violencia familiar. Índice conformado por reactivos que evalúan conductas de violencia familiar (gritar, humillar, golpear, etc.).

Consistencia interna: 0.72

Satisfacción familiar. Índice conformado por el promedio de los reactivos que evalúan la satisfacción con los padres, y con los miembros de la familia.

Familiar cercano en prisión. Ítem que explora si algún familiar cercano del joven se encuentra actualmente, o ha estado, en prisión.

Ocupación. Ítem que explora la ocupación del joven.

Relevancia de estudiar. Ítem que explora si el joven considera que realizar estudios vale la pena.

Escuela o Rezago escolar. Ítem que explora si el joven repitió algún curso

Trabajo escolar.

Satisfacción con la escuela o trabajo. Índice conformado po el promedio de los reactivos que evalúan la satisfacción con la escuela/trabajo, los maestros/jefes y el desempeño escolar/ laboral.

Actividades recreativas prosociales
Número de grupos recreativos en que participa. Índice compuesto por la cantidad de grupos (artístico, deportivo, religioso, etc.) de los que el joven manifiesta formar parte.
Numérica.

Mayores puntajes corresponden a mayor cantidad de apoyo y control por parte de los padres.

Numérica

Mayores puntajes indican mayor cantidad de conductas de convivencia positiva o negativa. Numérica

Mayores puntajes indican mayor cantidad de violencia familiar. Numérica.

Mayores puntajes indican una mayor satisfacción.

Nominal: Sí o No

Nominal: Estudia, trabaja, ambos o ninguna.

Nominal: Sí o No Ordinal.

Ninguno; uno; y dos o más.

Numérica.

Mayores puntajes indican una mayor satisfacción.

Numérica.

Mayores puntajes indican mayor cantidad de grupos recreativos en los que participa. 


\section{Procedimiento.}

Se utilizó la información recolectada por el Instituto Nacional de Estadística y Geografía (Inegi) mediante la Ecopred y fueron seleccionadas únicamente las variables de interés que evaluaban cada factor del modelo de Andrews y Bonta, cuya pertinencia se determinó a partir de un análisis realizado por tres jueces con formación en psicología jurídica.

Posteriormente una regresión logística binaria fue realizada utilizando como variable de respuesta haber tenido o no un arresto policial en lo que va del año. Inicialmente se consideró incluir la variable sexo como una variable de control debido a que esta variable ha sido frecuentemente asociada de forma significativa con la conducta antisocial, por lo que se incluyó en el modelo para eliminar su efecto en otras variables. Todas las demás variables fueron añadidas en ocho bloques, cada uno de ellos correspondiente a los factores considerados en el modelo de Andrews y Bonta. El supuesto de momios proporcionales fue explorado a través del gráfico de residuos predichos versus probabilidad pronosticada, lo que confirmó el supuesto. Posteriormente, la aportación de cada bloque (factor) al modelo general fue determinada, así como también la significancia de cada indicador que conformó los bloques.

Finalmente, mediante el método de eliminación con pasos sucesivos hacia atrás, se buscó simplificar el modelo para conservar solamente aquellas variables que resultaran significativas para predecir los arrestos. Siguiendo el principio de parsimonia se obtuvo un modelo final con la menor cantidad de variables posibles y se guardaron las probabilidades pronosticadas con la finalidad de evaluar la adecuación del modelo resultante mediante los índices de especificidad y sensibilidad, así como con empleando el área por debajo de la Curva ROC.

Todos los análisis fueron realizados utilizando el software SPSS versión 24, a excepción de los índices de especificidad y sensibilidad que fueron obtenidos por medio del paquete JASP versión 0.8.4. Para todos los análisis un nivel de significancia de $\mathbf{0 . 0 1}$ fue empleado teniendo en consideración del tamaño grande de la muestra utilizada.

\section{Resultados}

Con el fin de analizar cuales indicadores y factores permitían determinar de forma significativa la presencia o ausencia de arrestos actuales, se llevó a cabo una regresión logística agrupando cada indicador en ocho bloques diferentes, cada uno refiriendo a uno de los ocho factores propuestos por el modelo de Andrews y Bonta. El modelo completo (incluyendo todos los indicadores y factores) tuvo un ajuste adecuado al modelo de regresión logística según la prueba de bondad de ajuste de Hosmer y Lameshow $\left(X^{2}=10.42, g l=8, p=.237\right)$; con una varianza explicada del $\mathbf{2 3 . 4 \%}$ según el pseudo- $R^{2}$ de Nagelkerke.

El modelo inicial sólo incluía la variable sexo como variable de control y esta probó ser significativa $\left(X^{2}=124.17, g l=1, p<.001\right)$, lo que indica que el sexo se encuentra estadísticamente asociado con los arrestos actuales. Según los estadísticos descriptivos el $\mathbf{8 4 . 1 \%}$ de los participantes quienes presentaron un arresto actual eran hombres, resultado que confirma el acierto al haber considerado el sexo como una variable de control en el modelo inicial.
Acto seguido se añadieron los ocho bloques (factores) para analizar su aporte en la predicción de los arrestos actuales. Tal como se aprecia en la Tabla 2, únicamente el factor de pensamientos pro-criminales y actividades recreativas prosociales no estuvieron asociados de forma significativa con los arrestos actuales. Por su parte, tanto el historial delictivo, la personalidad antisocial, la asociación delictiva, el abuso de sustancias, las relaciones familiares y la escuela o trabajo aportaron de forma significativa para predecir los arrestos con un nivel de significancia menor a $\mathbf{. 0 1}$.

Tabla 2 Aporte de cada factor del modelo de Andrews y Bonta (2010) a la predicción de los arrestos actuales

$\begin{array}{cccc}\text { Bloque (Factor) } & \text { Ji cuadrada } & \text { g.l. } & \text { Sig. } \\ \text { Historial delictivo } & 234.174 & 2 & p<.001 \\ \text { Personalidad antisocial } & 19.252 & 1 & p<.001 \\ \text { Pensamientos pro-criminales } & 2.370 & 2 & p=.306 \\ \text { Asociación delictiva } & 38.762 & 3 & p<.001 \\ \text { Abuso de sustancias } & 118.191 & 1 & p<.001 \\ \text { Relaciones familiares } & 26.359 & 10 & p<.01 \\ \text { Escuela/Trabajo } & 22.290 & 6 & p<.01 \\ \text { Actividades recreativas prosociales } & 1.328 & 1 & p=.249 \\ & \text { Fuente: elaboración propia }\end{array}$

También se analizó de forma más detallada cuáles de los indicadores utilizados para cada factor resultaron significativos para predecir los arrestos actuales. Como se aprecia en la Tabla 3, resultaron significativos haber realizado vandalismo, tener pares antisociales, haber pertenecido a una pandilla o banda, la cantidad de sustancias consumidas, la violencia familiar y el rezago escolar. Todas ellas - $a$ excepción de haber pertenecido a una banda/pandilla- con una significancia menor a $\mathbf{0 . 0 1}$.

Los resultados señalan que respecto al factor historial de conducta violenta tener antecedentes de vandalismo aumenta las posibilidades de un arresto actual más de un $\mathbf{2 0 0 \%}(O R=2.34)$. Respecto al factor asociación criminal se encontró que tener mejores amigos que llevan a cabo conductas/actos antisociales aumenta la posibilidad de arrestos actuales de manera directamente proporcional, es decir, a mayor cantidad de conductas antisociales realizadas mayor probabilidad de ser arrestado. De igual forma, haber pertenecido a una banda o pandilla también aumenta las posibilidades de tener un arresto actual hasta un $\mathbf{7 0 \%}(O R=\mathbf{1 . 7 1})$. Finalmente, en contraste a lo anterior, no resultaron significativos los indicadores de los factores de personalidad antisocial ni de pensamientos pro-criminales.

Respecto a los factores moderadores, específicamente el factor de abuso de sustancias, se encontró una relación directamente proporcional, a mayor cantidad de sustancias experimentadas mayor posibilidad de tener un arresto actual. Respecto al factor relaciones familiares, se encontró que una mayor puntuación en el índice de violencia familiar se asocia con una mayor probabilidad de tener un arresto, aumentando hasta más de tres veces la posibilidad del arresto por cada punto adicional en el índice de violencia familiar (OR = 3.52). Con relación al factor de escuela/trabajo, el rezago educativo demostró estar estadísticamente asociado a los arrestos, al comparar un joven quien no haya repetido cursos con jóvenes quienes han repetido uno, o dos o más cursos, la posibilidad de tener un arresto actual aumenta un $\mathbf{8 1} \%$ y $\mathbf{9 7 \%}$ respectivamente $(O R=1.81 \& 1.97 \mathrm{c} / \mathrm{u})$. Por último, los indicadores pertenecientes al factor moderador actividades recreativas prosociales no resultaron significativos. 


\section{Ocho factores de la conducta criminal}

Aplicabilidad en jóvenes mexicanos

Tabla 3 Aporte de cada indicador en el modelo completo según los ocho factores propuestos por Andrews y Bonta

\begin{tabular}{|c|c|c|c|c|c|}
\hline \multirow{2}{*}{ Variable } & \multirow{2}{*}{ B } & \multirow{2}{*}{ Sig } & \multirow{2}{*}{ O.R. } & \multicolumn{2}{|c|}{ IC (95\%) } \\
\hline & & & & Inf & Sup \\
\hline Sexo (Referencia: hombre) & 1.360 & $p<.001$ & 3.895 & 2.684 & 5.652 \\
\hline \multicolumn{6}{|l|}{ Antecedentes delictivos } \\
\hline Realizó vandalismo & .852 & $p<.001$ & 2.344 & 1.616 & 3.400 \\
\hline Porta armas para defenderse & .089 & .694 & 1.093 & .703 & 1.700 \\
\hline \multicolumn{6}{|l|}{ Personalidad Antisocial } \\
\hline Impulsividad & -.111 & .366 & .895 & .703 & 1.138 \\
\hline \multicolumn{6}{|l|}{ Pensamientos pro-criminales } \\
\hline Admira a un criminal & -18.604 & 0.999 & .000 & .000 & \\
\hline $\begin{array}{c}\text { Admira a un jefe de una banda } \\
\text { violenta }\end{array}$ & 1.133 & .312 & 3.106 & .345 & 27.984 \\
\hline \multicolumn{6}{|l|}{ Asociaciones criminales } \\
\hline Pares Antisociales & .936 & .005 & 2.550 & 1.329 & 4.894 \\
\hline $\begin{array}{c}\text { Compañeros de trabajo/escuela con } \\
\text { actitudes antisociales }\end{array}$ & .222 & .390 & 1.249 & 0.752 & 2.073 \\
\hline Perteneció a una pandilla/banda & .537 & .015 & 1.711 & 1.110 & 2.638 \\
\hline \multicolumn{6}{|l|}{ Abuso de sustancias } \\
\hline $\begin{array}{l}\text { Cantidad de drogas que ha } \\
\text { experimentado }\end{array}$ & .524 & $p<.001$ & 1.689 & 1.511 & 1.888 \\
\hline \multicolumn{6}{|l|}{ Relaciones familiares } \\
\hline ¿Cómo te sientes en casa? & -.021 & .903 & .979 & .699 & 1.372 \\
\hline $\begin{array}{l}\text { Horas promedio de convivencia con } \\
\text { padres }\end{array}$ & -.015 & .518 & .986 & .943 & 1.030 \\
\hline Atención de la madre & .177 & .613 & 1.193 & .602 & 2.364 \\
\hline Control de la madre & -.003 & .992 & .997 & .570 & 1.744 \\
\hline Atención y control del padre & -.092 & .632 & .912 & .625 & 1.330 \\
\hline Convivencia familiar positiva & -.470 & .161 & .625 & .324 & 1.207 \\
\hline Convivencia familiar negativa & .137 & .582 & 1.147 & .704 & 1.866 \\
\hline Violencia Familiar & 1.261 & .004 & 3.527 & 1.494 & 8.327 \\
\hline Satisfacción familiar & -.061 & .667 & .941 & .713 & 1.242 \\
\hline Tuvo algún familiar cercano en prisión & .301 & .054 & 1.352 & .995 & 1.836 \\
\hline Escuela/Trabajo & \multicolumn{5}{|c|}{ Ocupación (Referencia: Estudia) } \\
\hline Ocupación: Trabaja & .323 & .063 & 1.381 & .982 & 1.941 \\
\hline Ocupación: Estudia y trabaja & .144 & .496 & 1.155 & .763 & 1.750 \\
\hline Considera que estudiar vale la pena & .110 & .814 & 1.117 & .445 & 2.802 \\
\hline \multicolumn{6}{|l|}{ Rezago escolar (¿Repitió un curso escolar?) } \\
\hline Uno & .594 & $p<.001$ & 1.811 & 1.339 & 2.449 \\
\hline Más de uno & .679 & .007 & 1.973 & 1.204 & 3.233 \\
\hline Satisfacción laboral/escolar & -.011 & .945 & .989 & .713 & 1.370 \\
\hline \multicolumn{6}{|l|}{ Actividades recreativas prosociales } \\
\hline $\begin{array}{l}\text { Cantidad de grupos prosociales en los } \\
\text { que participa }\end{array}$ & -.170 & .264 & .843 & .625 & 1.137 \\
\hline Constante & -5.691 & $p<.001$ & .003 & & \\
\hline
\end{tabular}

Nota: O.R. = Odds ratio o razón de momios; I.C. = Intervalo de confianza; Fuente: elaboración propia

Siguiendo el principio de parsimonia, se procedió a la simplificación del modelo mediante la técnica de pasos sucesivos hacia atrás. Tal como se observa en la Tabla 4, el modelo resultante consideró únicamente nueve variables, entre ellas la variable de control sexo. El modelo simplificado explicaba una varianza del $\mathbf{2 2 . 9 \%}$ según el pseudo- $R^{2}$ de Nagelkerke, muy cercano al modelo completo. Además, la prueba de bondad de ajuste de Hosmery Lameshow señala la adecuación del modelo $\left(X^{2}=16.14, g l=7, p=.024\right)$.

Tabla 4 Aporte de las variables resultantes del modelo simplificado

\begin{tabular}{|c|c|c|c|c|c|}
\hline \multirow{2}{*}{ Variable } & \multirow{2}{*}{ B } & \multirow{2}{*}{ Sig. } & \multirow{2}{*}{ OR } & \multicolumn{2}{|c|}{ IC $95 \%$} \\
\hline & & & & Inf & Sup \\
\hline Sexo (ser hombre) & -1.379 & $p<.001$ & .252 & .174 & .364 \\
\hline \multicolumn{6}{|l|}{ Antecedentes delictivos } \\
\hline Realizó vandalismo & .872 & $p<.001$ & 2.391 & 1.657 & 3.449 \\
\hline \multicolumn{6}{|l|}{ Asociaciones criminales } \\
\hline Pares Antisociales & 1.048 & .001 & 2.852 & 1.530 & 5.317 \\
\hline Perteneció a una banda & .545 & .010 & 1.725 & 1.138 & 2.615 \\
\hline \multicolumn{6}{|l|}{ Abuso de sustancias } \\
\hline $\begin{array}{l}\text { Cantidad de drogas que ha } \\
\text { experimentado }\end{array}$ & .564 & $p<.001$ & 1.758 & 1.587 & 1.946 \\
\hline \multicolumn{6}{|l|}{ Relaciones familiares } \\
\hline Convivencia familiar positiva & -.632 & .035 & .532 & .296 & .956 \\
\hline Violencia Familiar & 1.395 & .001 & 4.035 & 1.805 & 9.019 \\
\hline Tuvo algún familiar cercano en prisión & .320 & .040 & 1.376 & 1.015 & 1.866 \\
\hline \multicolumn{6}{|l|}{ Escuela o Trabajo } \\
\hline ¿Repitió un curso escolar? & & $p<.001$ & & & \\
\hline Uno & .637 & $p<.001$ & 1.891 & 1.403 & 2.549 \\
\hline Dos o más & .759 & .002 & 2.136 & 1.311 & 3.481 \\
\hline Constante & -4.572 & $p<.001$ & .010 & & \\
\hline
\end{tabular}

Nota: O.R. = Odds ratio o razón de momios; I.C. = Intervalo de Confianza; Fuente: Elaboración propia

Tal como se observa en la Tabla 4, todos los factores significativos identificados en el modelo completo fueron conservados; sin embargo, dos variables más fueron añadidas al modelo simplificado, las cuales resultaron significativas, a saber: convivencia familiar positiva y tener algún familiar cercano en prisión, a pesar que el nivel de significancia de ambas fue de $\mathbf{0 . 0 5}$.

La primera de estas variables resulta ser un factor de protección, a diferencia de las demás variables, pues mayor convivencia familiar positiva estuvo inversamente asociada a la posibilidad de tener un arresto en lo que va del año, disminuyendo hasta casi un $\mathbf{5 0 \%}$ esta posibilidad $(O R=0.53)$. Por su parte, tener un familiar cercano en prisión fue un factor de riesgo que aumentó casi un $\mathbf{4 0 \%}$ la posibilidad de tener un arresto actual $(O R=1.37)$.

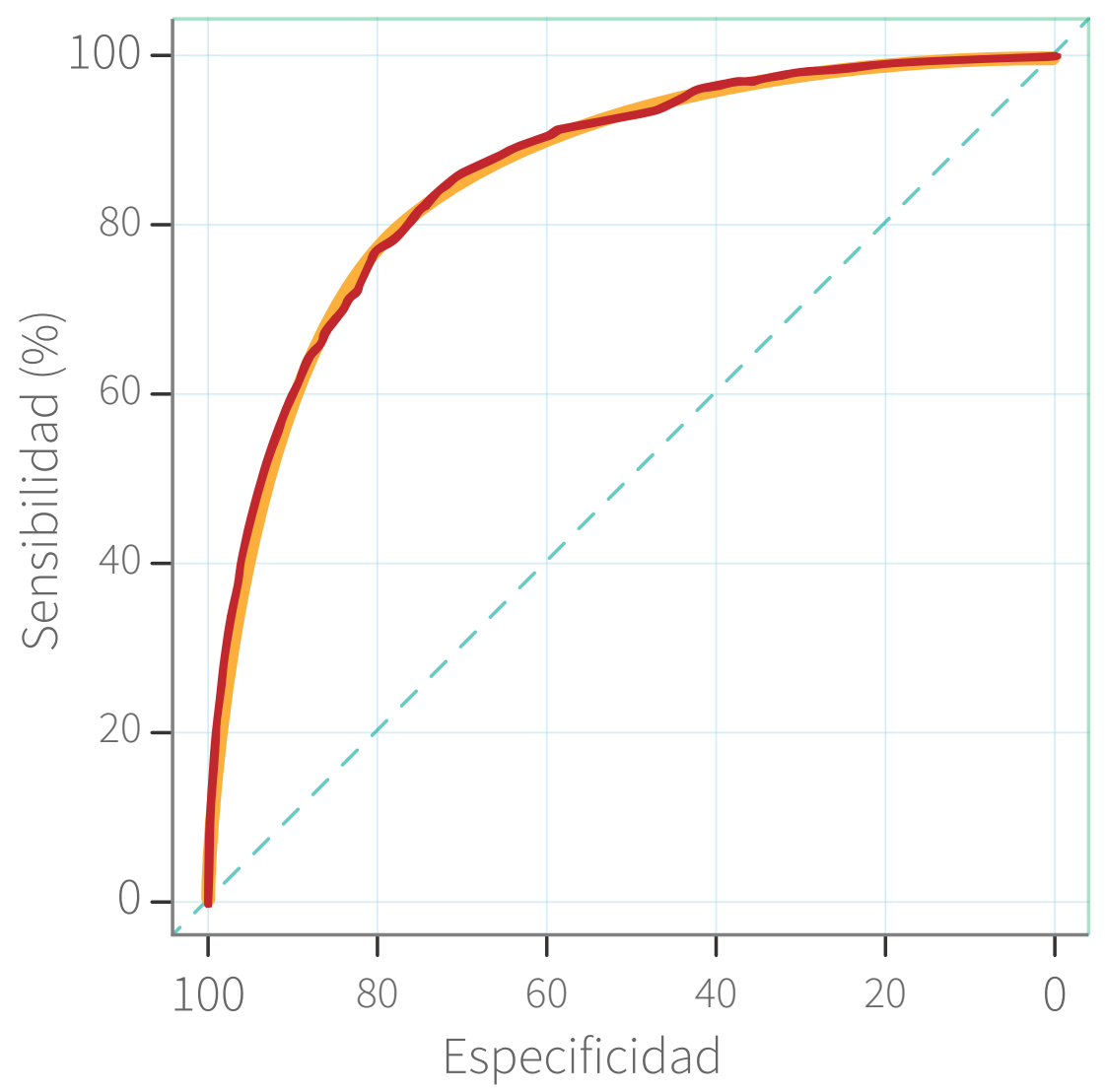

Gráfico 1 Curva ROC del modelo simplificado 
El modelo resultante, al igual que el modelo completo, sólo contempla dos de los cuatro grandes factores propuestos por Andrews y Bonta, a saber: antecedentes delictivos y asociación delictiva. Ni los pensamientos pro-criminales, ni la personalidad antisocial resultaron significativos. Asimismo, contempla tres de los cuatro factores moderadores: abuso de sustancias, relaciones familiares y educación/ trabajo, en tanto las actividades recreativas prosociales no resultaron significativas.

Finalmente, la adecuación del modelo final fue evaluada a través de los indicadores de especificidad, sensibilidad y el área bajo la Curva ROC. A partir de las puntuaciones pronosticadas por el modelo simplificado se obtuvo que éste tiene una 1-sensibilidad de $\mathbf{9 6 3}$ y una especificidad de.999. El área bajo de la Curva ROC, tal como se observa en la Figura 1, fue mejor que el azar, con un área bajo la curva de $\mathbf{. 8 6 8}$ (IC [95\%] = $0.852-0.884$ ), lo que indica que el modelo simplificado de nueve indicadores clasifica de forma adecuada a los jóvenes con arrestos recientes respecto a los que no tuvieron arrestos.

\section{Discusión}

En general, el estudio fue consistente con algunos resultados obtenidos en estudios previos, por ejemplo, se encontró que el historial de conducta antisocial, la asociación criminal, el abuso de sustancias, las pobres relaciones familiares y los problemas con el trabajo y la escuela están asociados con tener una mayor posibilidad de arresto actual, resultados acordes con investigaciones anteriores (Bertone, Domínguez, Vallejos, Muniello, \& López, 2013; Herrera, Rueda, \& Martínez, 2013; James, 2015; Martínez-Catena \& Redondo, 2013; Ortega Campos, García, de la Fuente Sánchez, \& Zaldívar Basurto, 2012). Esta consistencia sugiere que estos factores deberían ser considerados al realizar programas de prevención o de tratamiento penitenciario.

Respecto a los arrestos actuales, nuevamente se confirma la presencia de diferencias según el sexo de los participantes, siendo los hombres quienes presentan mayor posibilidad de ser arrestados que las mujeres. Este resultado es consistente con estudios previos que señalan la relación entre la delincuencia y el sexo, en los que hombres presentan mayor probabilidad de ser arrestados o reincidir (Cuervo, Villanueva, \& Prado-Gascó, 2017; Ortega Campos, García, de la Fuente Sánchez, \& Zaldívar Basurto, 2012).

Sin embargo, a diferencia de otros estudios, resultados inusuales fueron obtenidos en el presente. Si bien el modelo RNR propuesto por Andrews y Bonta manifiesta una significación muy consistente en cuanto al aporte de los factores propuestos (Brogan, Haney-Caron, NeMoyer, \& DeMatteo, 2015), lo cierto es que esto ocurre en muestras provenientes del contexto canadiense. En el presente estudio se encontró que la personalidad antisocial no fue un predictor para los arrestos, resultado obtenido en otros estudios realizados en contextos no-canadienses, como Australia (McGrath \& Thompson, 2012) o Alemania (Grieger \& Hosser, 2014). Los pensamientos pro-criminales tampoco resultaron significativos, sin embargo, otro estudio similar había encontrado resultados coincidentes en Singapur (Chu, Yu, Lee, \& Zeng, 2014). Finalmente, la nosignificancia de las actividades recreativas prosociales también parece ser un patrón que se da en otros países (Chu, Yu, Lee, \& Zeng, 2014; Grieger \& Hosser, 2014; McGrath \& Thompson, 2012), lo que indica que el poco aporte de este factor al modelo no es un fenómeno exclusivo de México.

La discrepancia entre estos resultados con la consistencia que ha tenido el modelo en general puede deberse a diversos factores. Inicialmente, el aspecto geográfico es uno de los factores que mayor variabilidad añaden a la consistencia del modelo (Olver, Stockdale, \& Wormith, 2014), y posiblemente se deba al efecto que genera la cultura sobre los diversos factores dinámicos del modelo propuesto por Andrews y Bonta, por ejemplo, los estilos de crianza de los padres y las actividades que se realizan como recreación en diversos países. Otro aspecto a considerar se relaciona con la influencia de la edad, pues existe evidencia sobre la importancia del desarrollo en la conducta delictiva, la cual sugiere que a medida que el adolescente crece los factores pueden variar o perder influencia, sobre todo si la persona no ha tenido un inicio temprano en actividades delictivas (Hoge, 2015).

Otra posible razón es que el instrumento empleado (Ecopred) para medir las variables de este estudio no fue expresamente diseñado para medir los ocho factores de Andrews y Bonta, y la mayoría de los estudios que evalúan dicho modelo toman en consideración instrumentos expresamente diseñados para evaluarlo (como el Level of Service o el Youth Level of Service (Cuervo, Villanueva, \& Prado-Gascó, 2017; Olver, Stockdale, \& Wormith, 2014)). Además, a diferencia de los estudios mencionados a lo largo de este trabajo, este estudio evalúa una muestra de jóvenes que no necesariamente presenta problemas con la ley, lo que posiblemente pueda afectar significación de variables importantes (como las actitudes pro-criminales o la personalidad antisocial) hacia su disminución.

En general, si bien el presente estudio señala que el modelo propuesto por Andrews y Bonta no se ajusta completamente a la realidad del contexto juvenil mexicano, lo cierto es que el ajuste presentado por el modelo obtenido es considerablemente bueno, pues sus índices de sensibilidad, especificidad y área bajo la Curva ROC son similares, e incluso mejores, a otros estudios previos que presentan valores cercanos a $\mathbf{0 . 7 5}$ a $\mathbf{0 . 8 3}$ (Andrews, y otros, 2012). El modelo obtenido presenta un área bajo la curva entre $\mathbf{8 5}$ y $\mathbf{8 8 \%}$, lo que indica que las variables identificadas en este modelo son significativamente mejores que el azar para predecir los arrestos en los jóvenes. Además, el modelo resultante explica casi un $\mathbf{2 3} \%$ de la varianza, mientras que otros modelos simplificados apenas explican poco más del $\mathbf{1 0} \%$ de la variación (McGrath \& Thompson, 2012). Por lo anterior se puede concluir que, aunque no todos los factores del modelo de Andrews y Bonta resultaron significativos en el contexto de México, ciertamente es un modelo que puede ayudar a explicar de forma apropiada la conducta criminal en México.

Finalmente, resulta preciso mencionar las limitaciones que presenta el éste estudio. Sibien la muestra grandeysu representatividad son una fortaleza, al menos tres grandes limitaciones deben ser consideradas. A nivel metodológico sobresale que se utilizó un instrumento que no fue diseñado para evaluar el modelo de Andrews y Bonta, lo que sin duda podría afectar la validez de algunos indicadores utilizados para evaluar los factores. Además, el instrumento utilizado fue una encuesta de autoreporte, por lo que la deseabilidad social podría ser un factor que influye en las respuestas de los jóvenes. Por lo anterior se recomienda realizar estudios similares en México, pero empleando instrumentos apropiados, diseñados según el modelo de Andrews y Bonta.

Por último, a nivel teórico hay que señalar que el modelo fue probado con muestras de jóvenes quienes no necesariamente tienen, o han tenido, algún problema con la ley -a diferencia de estudios internacionales que emplean este modelo para explicar, manejar y prevenir la delincuencia juvenil, especialmente la reincidencia juvenil-. Sin duda algún se recomienda que estudios futuros exploren este modelo en muestras de jóvenes que presenten problemas con la ley en México para así conocer el ajuste del modelo a este contexto. 


\section{Conclusión}

En conclusión, resulta sustancial realizar nuevas investigaciones que contribuyan a los resultados expuestos en el presente artículo, orientadas a explorar el Modelo Riesgo-Necesidad-Responsividad (RNR) en población con antecedentes delictivos, particularmente jóvenes con problemas con la ley.

Es importante continuar realizando de forma frecuente estudios como la Encuesta de Cohesión Social de la Violencia y la Delincuencia (Ecopred), pues su representatividad nacional proporciona un panorama general de la situación de la violencia y delincuencia en México, y analizar sus resultados a partir de modelos científicos ayuda a tener una visión más clara sobre cómo intervenir para mejorar la situación del país.

Hoy en día resulta imperante que en México se desarrolle política pública tomando en cuenta la evidencia científica, ya que esto ayudaría a evitar el uso de recursos económicos, personales y administrativos, en programas sin fundamento. Para disminuir la violencia y la delincuencia es conveniente que se desarrollen estrategias, proyectos y programas sociales que tomen en cuenta tanto indicadores con sustento científico -como los contemplados en este estudio- como estrategias orientadas a la intervención en temas como la prevención de consumo de sustancias, disminución del pandillerismo y fomento de amistades positivas, disminución del rezago escolar, promoción de la convivencia familiar positiva o, en contraparte, la disminución de la violencia familiar.

\section{Referencias}

Andrews, D. A., \& Bonta, J. (2010). The Psychology of Criminal Conduct (5 ed.). New Providence: Anderson Publishing. DOI:10.1017/CBO9781107415324.004

Andrews, D. A., Guzzo, L., Raynor, P., Rowe, R. C., Rettinger, L. J., Brews, A., \& Wormith, J. S. (2012). Are the Major Risk/Need Factors Predictive of Both Female and Male Reoffending? International Journal of Offender Therapy and Comparative Criminology, 113-133. DOI:10.1177/0306624X10395716

Assink, M., van der Put, C. E., Hoeve, M., Sanne de Vries, L. A., Stams Geert Jan, J. M., \& Oort Frans, J. (2015). Risk factors for persistent delinquent behavior among juveniles: A meta-analytic review. Clinical Psychology Review, 4761. DOI:10.1016/j.cpr.2015.08.002

Banse, R., Koppehele-Gossel, J., Kistemaker, L. M., Werner, V. a., \& Schmidt, A. F. (2013). Pro-criminal attitudes, intervention, and recidivism. Aggression and Violent Behavior, 673-685. DOI:10.1016/j.avb.2013.07.024

Benavides, A., Romero, M., Fernández, M., \& Pichardo, M. C. (2017). Deporte en familia como prevención de problemas de conducta desde edades tempranas. International Journal of Developmental and Psychology, 3 (1) 449-456. DOI:10.17060/ijodaep.2017.n1.v3.1014

Benedicto, C. (2015). Evolución de los factores de riesgo dinámicos en función del historial delictivo de menores infractores de la Comunidad de Madrid. Universidad Complutense de Madrid.

Bertone, M., Domínguez, M. S., Vallejos, M., Muniello, J., \& López, P. (2013). Variables asociadas a la reincidencia delictiva. Psicopatología Clínica, Legaly Forense, 13 (1) 47-58. Obtenido de: http://masterforense.com/ index.php?option=com content\&view $=$ article\&id $=87 \&$ ltemid $=161$

Boduszek, D., Hyland, P., Pedziszczak, J., \& Kielkiewicz, K. (2012). Criminal attitudes, recidivistic behaviour, and the mediating role of associations with criminal friends: An empirical investigation within a prison sample fiolent offenders. Europe's Journal of Psychology, 18-31. DOI:10.5964/ejop.v8i1.296

Bonta, J., \& Andrews, D. A. (2012). Vieweing offender assessment and rehabilitation through the lens of the risk-need-responsivity model. New York [us]: Routledge.

Brogan, L., Haney-Caron, E., NeMoyer, A., \& DeMatteo, D. (2015). Applying the Risk-
Needs-Responsivity (RNR) Model to Juvenile Justice. Criminal Justice Review, 277-302. DOI:10.1177/0734016814567312

Chu, C. M., Yu, H., Lee, Y., \& Zeng, G. (2014). The Utility of the YLS/CMI-SV for Assessing Youth Offenders in Singapore. Criminal Justice and Behavior, 1437-1457. DOI:10.1177/0093854814537626

Contreras Martínez, L., Molina Banqueri, V., \& Cano Lozano, M. C. (2012). Consumo de drogas en adolescentes con conductas infractoras: Analisis de variables psicosociales implicadas. Adicciones, 31-38. DOI:10.20882/ adicciones. 115

Cuervo, K., Villanueva, L., \& Prado-Gascó, V. (2017). Predicción de la reincidencia juvenil mediante el YLS/CMI y baremos para su valoración. Revista Mexicana de Psicología, 34 (1) 24-36.

Dawes, R. M., \& Corrigan, B. (1974). Lineal models in decision making. Psychological Bulletin, 95-106. DOI:10.1037/h0037613

Farrington, D. P., Gaffney, H., \& Ttofi, M. M. (2017). Systematic reviews of explanatory risk factors for violence, offending, and delinquency. Aggression and Violent Behavior, 24-36. DOI:10.1016/j.avb.2016.11.004

Grieger, L., \& Hosser, D. (2014). Which Risk Factors are Really Predictive?: An Analysis of Andrews and Bonta's "Central Eight" Risk Factors for Recidivism in German Youth Correctional facility Inmates. Criminal Justice and Behavior, 613-634. DOI:10.1177/0093854813511432

Herrera, M. E., Rueda, A. A., \& Martínez, L. R. (2013). Factores de riesgo que identifican a adolescentes y jóvenes en conflicto con la ley. Psicología y Salud, 209-216.

Hoge, R. (2015). Risk, Need and Responsivity in Juveniles. [us]: APA, American Psychological Association.

Inegi. (2014). Encuesta de Cohesión Social para la Prevención de la Violencia y la Delincuencia: Presentación Ejecutiva. México DF [mx]: Inegi, Instituto Nacional de Estadística y Geografía.

Inegi. (2016). Censo Nacional de Impartición de Justicia Estatal 2016. México DF [mx]: Inegi, Instituto Nacional de Estadística y Geografía.

James, N. (2015). Risk and Needs Assessment in the Criminal Justice System. [us].

Jolliffe, D., Farrington, D. P., Piquero, A. R., Loeber, R., \& Hill, K. G. (2017). Systematic review of early risk factors for life-course-persistent, adolescencelimited, and late-onset offenders in prospective longitudinal studies. Aggression and Violent Behavior, 15-23. DOI:10.1016/j.avb.2017.01.009

Looman, J., \& Abracen, J. (2013). The risk need responsivity model of offender rehabilitation: Is there really a need for a paradigm shift? International Journal of Behavioral Consultation and Therapy, 30-36. DOI:10.1037/h0100980

Martínez, I., Fuentes, M., García, F., \& Madrid, I. (2013). El estilo de socialización familiar como factor de prevención o riesgo para el consumo de sustancias y otros problemas de conducta en los adolescentes españoles. Adicciones, 235-242. DOI:10.20882/adicciones.51

Martínez-Catena, A., \& Redondo, S. (2013). Carreras delictivas juveniles y tratamiento. Zerbitzuan, 171-183. DOI:10.5569/1134-7147.54.12

McGrath, A., \& Thompson, A. P. (2012). The Relative Predictive Validity of the Static and Dynamic Domain Scores in Risk-Need Assessment of Juvenile Offenders. Criminal Justice and Behavior, 250-263. DOI:10.1177/0093854811431917

Montedónico Godoy, S. (2015). Una mirada a los discursos sobre Desistimiento del delito en jóvenes que se encuentran cumpliendo una sanción penal. Universidad de Chile.

Moreira Trillo, V. (2011). Grupo de amigos, Género y Delincuencia Juvenil. Universidad de Santiago de Compostela.

Navarro-Pérez, J. J., \& Pastor-Seller, E. (2017). Factores dinámicos en el comportamiento de delincuentes juveniles con perfil de ajuste social. Un estudio de reincidencia. Psychosocial Intervention, 19-27. DOI:10.1016/i.psi.2016.08.001

Nguyen, T., Arbach-Lucioni, K., \& Andrés-Pueyo, A. (2011). Factores de riesgo de la reincidencia violenta en población penitenciaria. Revista de Derecho Penaly Criminología, 3 epoca (6) 273-294

Olver, M. E., Stockdale, K. C., \& Wormith, J. S. (2014). Thirty years of research on the Level of Service Scales: A meta-analytic examination of predictive accuracy and sources of variability. Psychological Assessment, 156-176. DOI:10.1037/a0035080

Ortega Campos, E., García, J. G., de la Fuente Sánchez, L., \& Zaldívar Basurto, F. (2012). Meta-análisis de la reincidencia de la conducta antisocial penada en adolescentes españoles. EduPsykhé: Revista de Psicología y Educación 11 (1) 171-189. 
Ortega-Campos, E., García-García, J., \& Frías-Armenta, M. (2014). Meta-análisis de la reincidencia criminal en menores: estudio de la investigación española. Revista Mexicana de Psicología, 31 (2) 111-123.

Pelegrín, A., \& Garcés de los Fayos, E. (2008). Variables contextuales que inciden en el comportamiento violento del niño. European Journal of Education and Psychology, 1 (1) 5-20. DOI:10.30552/ejep.v1i1.1

Pérez-Luco, R., Lagos, L., \& Báez, C. (2012). Reincidencia y desistimiento en adolescentes infractores: Análisis de trayectorias delictivas a partir de autorreporte de delitos, consumode sustancias y juicio profesional. Universitas Psychologica, 11 (4) 1209-1225.

Rodriguez, J. A. (2015). Un análisis de la relación entre grupo de amigos, edad y conducta antisocial: Delimitando diferencias de género. Archivos de criminología, seguridad privada y criminalística, IV (2) 1-20.

Rodríguez, J. A., Mirón, L., \& Rial, A. (2012). Análisis de la relación entre grupo de iguales, vinculación familiar y escolar, autocontrol y conducta antisocial, en una muestra de adolescentes venezolanos. Revista de Psicologia Social, 25-38. DOI:10.1174/021347412798844033

Rojas Zegarra, E. M. (2013). Distorsiones cognitivas y conducta agresiva en jóvenes y adolescentes: Análisis en muestras comunitarias y de delincuentes. Universidad Complutense de Madrid.

Schioppa, M. (2013). Influencia del grupo de Pares delincuentes en la implicación Criminal pairs and the influence in delinquency adolescent involvement.
Justicia Juris, 93-111. DOI:10.15665/ri.v9i1.102

Thompson, K. C., \& Morris, R. J. (2013). Predicting Recidivism Among Juvenile Delinquents: Comparison of Risk Factors for Male and Female Offenders. Journal of Juvenile Justice, (3) 36-47.

Unodc. (2010). La Relación Droga y Delito en Adolescentes Infractores de la Ley: La experiencia de Bolivia, Chile, Colombia, Perú y Uruguay [Quinto Informe Conjunto]. Ginebra [ch]: Unodc, United Nations Office on Drugs and Crime [Oficina de las Naciones Unidas contra la Droga y el Delito].

Unodc. (2013). Guía de Introducción a la Prevención de la Reincidencia y la Reintegración Social de Delincuentes. Ginebra [ch]: Unodc, United Nations Office on Drugs and Crime [Oficina de las Naciones Unidas contra la Droga y el Delito].

Velásquez Valenzuela, J. E. (2014). El origen del paradigma de riesgo. Politica Criminal, 58-117. DOI:10.4067/S0718-33992014000100003

Vilariño, M., Amado, B. G., \& Alves, C. (2013). Menores infractores: un estudio de campo de los factores de riesgo. Anuario de Psicología Jurídica, 39-45. DOI:10.5093/aj2013a7

Wilson, H., \& Hoge, R. (2013). Diverting our attention yo what Works: evaluating the effectiveness of a youth diversion program. Youth Violence and Juvenile Justice, 11 (4) 313-331. DOI:10.1177/1541204012473132

de Foco Rojo: Centro de Psicología Aplicada. Trabajó en el Centro Especializado en la Aplicación de Medidas para Adolescentes del estado de Yucatán en el departamento de psicología. Sus líneas de investigación son adolescentes en conflicto con la ley, prevención de la violencia, psicología jurídica, psicología forense, Derechos Humanos y perfilación criminal.

$\mathrm{BIO}$ :

Obutvo el título de Licenciado en Psicología por la Universidad Autónoma de Yucatán (UADY), y posteriormente curso la Especialización en Estadística por la Facultad de Matemáticas de la misma casa de estudios. Actualmente, se encuentra finalizando la Maestría en Investigación Educativa en la Facultad de Educación de la UADY. Ha participado en proyectos de prevención social de la violencia y la delincuencia tanto de forma externa como por parte de la Secretaría de Seguridad Pública, principalmente en el diseño e implementación de encuestas de victimización. Ha colaborado con el Grupo de Estudios Avanzados en Violencia de la Universidad de Barcelona realizando modelos predictivos de reincidencia delictiva, y actualmente es colaborador externo en el Instituo Nacional de Psiquiatría "Ramón de la Fuente Muñiz" en temas de adicciones. Entre sus temas de interés de investigación se encuentra la reincidencia delictiva, la valoración del riesgo de violencia, las encuestas de victimización, el acoso escolar y las adicciones.

Lic Graciela Mayte Chale Cervantes, MSc Psi

ORCID:*0000-0003-1042-4475

$\mathrm{BIO}$ :

Maestra en psicología aplicada en el área de la criminología por la Universidad Autónoma de Yucatán (UADY) y becada por el CONACYT. Ganadora del premio a mejor trabajo de titulación otorgada por la Comisión de Derechos Humanos de Yucatán y el Colegio de Abogados de Yucatán con la tesis "Intervención para el desarrollo de habilidades sociales en un adolescente en conflicto con la ley". Como parte de su formación, realizó una estancia de investigación en la facultad de psicología de la Universidad Complutense de Madrid. Se título de la licenciatura de psicología por la UADY con el artículo "Modelos de prevención de riesgo de violencia auto infringida en una población de policías". Actualmente es Jefa de Monitoreo y Evaluación del Programa de Reinserción Social en Centros de Internamiento para Adolescentes de Reinserta un Mexicano A.C., donde trabaja con adolescentes en conflicto con la ley del Estado de México y la Ciudad de México. También es Socia y fundadora
Lic Aaron Javier Euan Catzin, Psi sp

ORCID:**0000-0001-6119-0352

$\mathrm{BIO}$ :

Licenciado en psicología por la Universidad Autónoma de Yucatán (México). Es socio fundador del Centro de Psicología Aplicada Foco Rojo, donde actualmente se desempeña como coordinador de evaluación psicológica. Principalmente se ha desempeñado coordinando proyectos sociales para la prevención de la violencia con adolescentes y jóvenes; así como en la coordinación de capacitación para elementos policiales. También ha colaborado en proyectos de diagnóstico local en temas de violencia y seguridad. Su área de investigación se enfoca en temas orientados a la prevención de la violencia.

Lic Cindy Carolina Cauich Sonda, MSc Psi ORCID: ${ }^{* * 0000-0003-0447-6658}$

$\mathrm{BIO}$ :

Maestra en Psicología Aplicada en el área Criminológica por la Universidad Autónoma de Yucatán (UADY), licenciada en psicología por la misma universidad, donde fue becaria del Programa de Impulso y Orientación a la Investigación (PRIORI) de tesis de licenciatura y becaria de Consejo Nacional para la Ciencia y Tecnología (CONACYT) en la maestría. Se ha desempeñado como docente en diversas universidadades de Yucatan, trabajó en la coordinación de programas de prevención social de la violencia, fue técnica de investigación en el Centro de Investigación de Psicologia Forense para Menores de la Faculdad de Psicología de la UADY. Actualmente laborá como Psicologa Forese en una en el Centro de Jisticia para las Mujeres del Estado de Yucatán, sus lineas de investigación son sobre violencia familiar, psicopatía, ética y ley, psicología forense, entre otras. Colaboradora Externa en Foco Rojo: Centro de Psicología Aplicada.

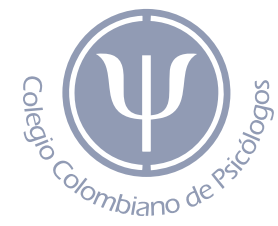




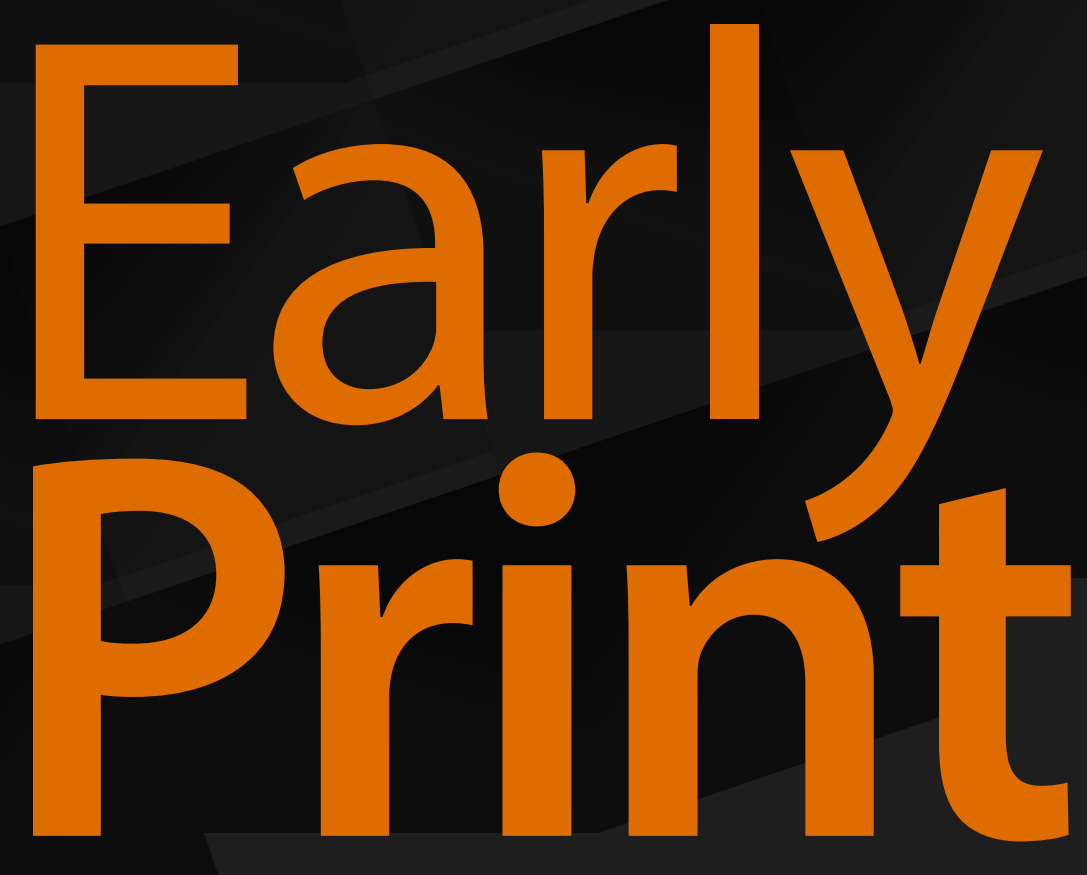

Desde el 1 de septiembre de 2018, todo artículo que sea

\section{ACEPTADO}

por los evaluadores y cuente con su documentación completa será

\section{PUBLICADO inmediatamente,}

así no deberá esperar a la fecha de publicación del número entero y el artículo estará disponible para el uso y consumo de la comunidad académica, científica y disciplinar.

Consulte las instrucciones para autores haciendo click aquí.

Mayor información:

revistaiberoamericana@ibero.edu.co

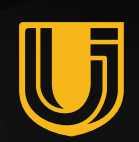

IBEROAMERICANA CORPORACIÓN UNIVERSITARIA
Revista Iberoamericana de
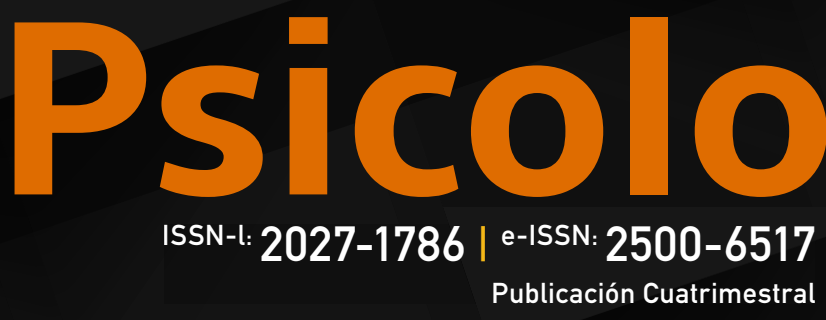